\title{
Form, Macht und Differenz als Dimensionen kultureller Praxis
}

\author{
Michael Dickhardt, Karin Klenke und Elfriede Hermann
}

Kulturelle Formen inmitten von Machtverhältnissen mit Blick auf Differenz zu untersuchen ist das Ziel eines Großteils ethnologischen Forschens. Form, Macht und kulturelle Differenz eröffnen ethnologische Felder für eine Vielzahl von Fragen - Fragen, die sich auch und gerade durch jene Bereiche ziehen, in denen das Forschen Brigitta Hauser-Schäublins sich vollzog und vollzieht, in denen es seine Motivation und seine vielfältigen Motive fand und findet: von Genderstudien zu den kulturellen Dimensionen der Körperlichkeit, von der materiellen Kultur zum musealen Objekt, von der Architektur zur kulturellen Räumlichkeit, vom Ritual zur Staatskultur, von der Dorfstudie zu den Verflechtungen der globalisierten Welt. In all diesen Feldern tauchen Form, Macht und kulturelle Differenz als Motiv immer wieder auf, doch sie tun dies nicht in einer klar bestimmbaren Konfiguration. ${ }^{1}$ Form, verstanden als die innere Fügung und die äußere Gestalt, Macht, verstanden als äußerliches restriktives Instrument und innerliches produktives Strukturierungsprinzip, und kulturelle Differenz, verstanden als identitätsstiftendes Moment und als identitätspolitische Ressource, gehen in und durch ihre Polyvalenzen immer wieder neue Verbindungen miteinander ein, die eine Vielfalt von Fragen aufwerfen: Lässt sich behaupten: Form macht Differenz? Oder lässt sich zeigen: Kulturelle Form und Macht begründen Differenz? Oder ist zu erkennen: Kulturelle Form und Macht begründen Differenz, Ähnlichkeit und/oder Gleichheit? Diese knapp formulierten Fragen können weiter ausgeführt werden: Welche Zusammenhänge bestehen zwischen dem Erschaffen kultureller Formen, Machtbeziehungen 
und der Herstellung von Differenzen, Ähnlichkeiten und Gleichheiten? Inwiefern sind Formen sowie kulturell geschaffene Differenz und/oder Gleichheit Ausdruck der Ausübung von Macht und Gegenmacht? Inwiefern wird Machtausübung durch kulturelle Formen und Differenzen beziehungsweise Gleichheiten ermöglicht oder verhindert? Inwiefern begründen kulturelle Formen in spezifischen Machtverhältnissen Differenz, Ähnlichkeit oder Gleichheit? Mit diesen Fragen ist eine für das ethnologische Interesse entscheidende Perspektive verknüpft: Inwiefern lassen kulturelle Formen in gegebenen Machtfeldern und Manifestationen von Differenz und/oder Gleichheit auf das Handlungsvermögen von sozialen Akteuren ${ }^{2}$ schließen?

Die hier zusammengetragenen Texte stammen nun aus ganz verschiedenen Feldern ethnologischen Forschens, in denen sich die genannten Fragen zum Zusammenhang von Form, Macht und Differenz stellen. Dabei war die Zusammenstellung der Texte von Beginn an nicht auf eine einheitliche thematische, theoretische oder methodologische Perspektive hin ausgerichtet. Vielmehr sollte die große Bandbreite ethnologischen Forschens sichtbar gemacht werden, die aber trotz ihrer Vielfältigkeit und Vielgestaltigkeit zusammengeführt wird durch jene spezifische ethnologische Perspektive, die das Werk Brigitta Hauser-Schäublins prägt. Diese besondere Perspektive wird weniger durch Begriffe wie Form, Macht und Differenz an und für sich ausgedrückt, als vielmehr durch die besondere Art, wie diese Begriffe in ihrer Relation zueinander bestimmt werden. Grundlegend für diese ethnologische Perspektive sind nun verschiedene Momente: die Anerkennung der Diversität kultureller und gesellschaftlicher Formen als Ausgangspunkt des verstehenden Erklärens menschlicher Praxis, die empirische Begründung in der Feldforschung im Modus der teilnehmenden Beobachtung, die kulturvergleichende Betrachtung und die methodische Nutzung der Fremdheitserfahrung. Alle diese Momente fußen aber auf einer zentralen Grundannahme, nämlich, dass menschliche Praxis von zwei untrennbar miteinander verbundenen Dimensionen gekennzeichnet wird - von der Objektivität der kulturellen Manifestationen und von der Subjektivität der Menschen, die diese Manifestationen in ihrem und durch ihr Handeln hervorbringen. Wie bestimmen sich also die Begriffe von Form, Macht und Differenz in einer ethnologischen Perspektive? Wie können die beiden wesentlichen Dimensionen soziokultureller Praxis ethnologisch bedeutsam unter der Perspektive des Zusammenhangs von Form, Macht und Differenz herausgearbeitet werden und zum Verstehen menschlicher Praxis beitragen?

Wenden wir uns zunächst dem Begriff der Form zu. Eingangs wurde er in seiner doppelten Bestimmung eingeführt: als innere Fügung und äußere Gestalt. ${ }^{3}$ Dies mag zunächst verwundern, hebt doch der übliche Sprachgebrauch innerhalb der Ethnologie sowie der Kultur- und Sozialanthropologie oft einseitig auf die äußere Gestalt ab. Doch ist der Begriff der Form nicht auf die äußere Gestalt eines hervorgebrachten kulturellen Gegenstandes zu reduzieren, sondern verweist immer auch auf die Art des Gestaltens, auf das Formen, das sich gesellschaftlich bestimmten Regeln der Bedeutungszuschreibung unterwirft. Diese haben sich wiederum zu gesellschaftlich anerkannten Arten der 
Strukturierung wie Sprache und Mythos, wie Rituale, Theater oder Literatur, wie Verwandtschaftsterminologien und Rollenvorstellungen kristallisiert. Vor diesem Hintergrund ist die Objektivität der äußeren Form immer Resultat eines Formungsprozesses, der sich in der Umsetzung der in den Regeln der Bedeutungszuschreibung angelegten Formungsprinzipien vollzieht. Und hier gelangt man zur inneren Fügung, denn das, was man als äußere Form erkennt, geht letztlich auf jene inneren Regeln und Prinzipien zurück, die bedeutungstragende Elemente wie Worte, Gesten oder materielle Objekte zusammenfügen mit den Bedeutungen, auf die sie verweisen. Das Äußere der Form und sein möglicher Bedeutungsgehalt ergeben sich nur in und durch diese Fügung, die in den Regeln und Prinzipien der Bedeutungszuschreibung angelegt ist und die sich durch den Formungsprozess konkretisiert. Hier nun freilich lässt die ethnologische Perspektive die Frage danach stellen, wie dieser Formungsprozess praktisch vonstatten geht, eine Frage, die uns zur Subjektivität der Menschen leitet, die die Formungen praktisch hervorbringen. Das Verhältnis der Akteure zu den soziokulturellen Formen ist nicht auf eines der bloßen Interpretation zu reduzieren. Wie gesagt, die Objektivität der Formen als äußere Gestalt entsteht durch einen Prozess der Formung, in dem Regeln und Prinzipien der Bedeutungszuschreibung und Bedeutungsverknüpfung materiale Bedeutungsträger mit Bedeutungen zusammenfügen. Dieses Fügen geschieht aber nicht von selbst, es geschieht durch die praktische Tätigkeit der Akteure. Diese deuten die äußeren Formen nicht bloß, sondern setzen diese praktisch um. Form erscheint somit als überaus komplexer Begriff, der es uns aus ethnologischer Perspektive erlaubt, die unterschiedlichen Dimensionen soziokultureller Praxis zu erfassen als die innere und die äußere Form und als das Formen in und durch die praktische Tätigkeit des Akteurs, als geformt und formend.

So vielschichtig das Feld auch sein mag, das der Begriff der Form einer ethnologischen Perspektive öffnen mag, so nachdrücklich haben die Debatten in der Ethnologie seit dem Einsetzen der postmodernistischen Kritik einen Begriff ins Zentrum der Aufmerksamkeit gerückt, ohne den keine Betrachtung der Form mehr auskommen kann: den Begriff der Macht. Es hat sich erwiesen, dass jeder Versuch, die Logik der soziokulturellen Formen nur aus sich selbst heraus zu verstehen, deren praktische Funktionen nicht erfassen kann - praktische Funktionen, die sich immer auch in einem von Macht bestimmten Feld entfalten. ${ }^{4}$ Wie aber wird Macht für die soziokulturellen Formen relevant? Am auffälligsten wird die Rolle der Macht, wenn die soziokulturellen Formen instrumentell eingesetzt werden, um Interessen der Akteure durchzusetzen. Der Mythos kann zur Legitimation von Herrschaft verwendet werden, das Ritual zur Reproduktion von Hierarchie. Doch, so warnt Sahlins, die Machtanalyse darf nicht dazu führen, die spezifische Logik der soziokulturellen Formen auszublenden oder auf eine bloße Funktion funktionalistisch-instrumentell interpretierter Machteffekte zu reduzieren (Sahlins 1999). Erst ein tiefes Verständnis kultureller Formen wie der des japanischen Sumo-Ringens erschließt ein Verständnis möglicher instrumenteller Machteffekte: 
Sumo can serve venal interests, thus enter into entrepreneurial projects, because of its meanings, associations and relationships in history and the culture. Function must be the servant of custom if it schemes to be its pragmatic master. (Sahlins 1999:409)

Doch ist die Beziehung zwischen function und custom, zwischen Macht und Form komplizierter als Sahlins es hier suggeriert. Die Form, so muss mit einem Foucault'schen cetero censeo eingewendet werden, entsteht nicht losgelöst von der Macht, um erst später von dieser funktionalisiert zu werden. Die Macht bestimmt den Kontext, in dem sich die Form allererst formieren kann, und sie nistet sich über Regeln und Prinzipien der Bedeutungszuschreibung und Bedeutungsverknüpfung in die Form ein: Die Bedeutungen des Sumo-Ringens konnten nur entstehen, indem sich das Sumo-Ringen durch die Übernahme bestimmter Formen wie zum Beispiel von Shinto-Ritualen und kosmologischer Symbolik in bestimmte Machtstrukturen einfügte. Dieser bedurfte es zu seiner Legitimation und half, sie wiederum selbst zu legitimieren, indem es allein durch die formende Fügung der Bedeutungen jene Bedeutungen hervorbringt, die die herrschende Ordnung in ihrer religiösen und kosmologischen Legitimation bestätigen. Form, so könnte man sagen, wird also nicht bloß funktionalisiert, sondern sie wird gemacht und macht selbst die Ordnung, innerhalb derer sie funktionalisiert wird, indem sie machtrelevante Bedeutungszuschreibungen und Bedeutungsverknüpfungen ermöglicht - und auch jene verhindert, die der Macht abträglich wären.

Eines der interessantesten Momente, das bei der Analyse des Zusammenhangs von Macht und Form immer wieder diskutiert wurde, ist das der Differenz. ${ }^{5}$ Differenz hat in der Geschichte der Ethnologie von Beginn an eine zentrale Rolle gespielt - und dies in unterschiedlichen epistemologischen, methodologischen und gegenstandstheoretischen Dimensionen, als gegebenes Faktum soziokultureller Praxis ebenso wie als Prinzip, dessen sich die Akteure bedienen, wenn sie vermittels der kulturellen und gesellschaftlichen Formen kulturelle Ordnungen aufbauen. Zunächst erscheint als wesentliches epistemologisches und methodologisches Moment der ethnologischen Perspektive eine doppelte Anerkennung der Differenz als Diversität kultureller und gesellschaftlicher Formen: zum einen als Ausgangspunkt des verstehenden Erklärens und zum anderen als methodologischer Rahmen, in dem Fremdheit kein Hindernis, sondern in ihrer Relationalität Voraussetzung ethnologischer Erkenntnis ist. ${ }^{6}$ Auf dieser Grundlage wurde die Differenz vielfältig in der Ethnologie thematisiert: als Explanans im Rahmen der Kulturbeschreibung und des Kulturvergleichs, als Explanandum im Verhältnis zur Idee der Einheit der Menschheit und als Strukturierungsprinzip von Bedeutungen im Sinne von Systemen von Unterschieden. Dabei wurde lange Zeit die Differenz zwischen Kulturen in den Vordergrund gerückt, was unter anderem zur Folge hatte, dass die Momente der Gleichheit und der Homogenität innerhalb der voneinander differenzierten Einheiten betont wurden - zu Lasten, wie sich bald herausstellte, einer angemessenen Repräsentation der Vielschichtigkeit und Widersprüchlichkeit soziokultureller Praxis. Die Reflexion der Differenz sowie ihrer Bedingungen, Funktionen und Implikationen im Forschungsprozess und in der soziokulturellen Praxis 
haben es dann möglich gemacht, die Logik ethnologischer Objektivierung und die Logik der Praxis in deren Komplexität und Heterogenität klarer und deutlicher hervortreten zu lassen. ${ }^{7}$ Differenz wurde nicht nur als gegebenes Faktum oder als Kontext soziokultureller Praxis, sondern zunehmend auch als Prinzip der Formung dieser Praxis durch die Akteure verstehbar. Die epistemologische und methodologische Kritik der Feldforschung und ihrer Formen der Repräsentation des Anderen erkannte in der Subjektivität des Forschers nicht mehr nur den privilegierten neutralen Ort der Erkenntnis, sondern eine im Erkenntnisprozess hervorgebrachte positionierte Perspektivität, die bestimmt wird durch komplexe Prozesse der Differenzierung, in denen der Feldforscher vermittels differenzierender Momente wie Hierarchie, Gender oder Alter $\mathrm{zu}$ einem konkreten sozialen, politischen, ökonomischen und ideologischen Akteur in praktischer Interaktion mit seinem Forschungsfeld und den ,beobachteten Akteuren wird. ${ }^{8}$ Die Ethnizitätsforschung hat gezeigt, dass es die Logik der Differenz und Abgrenzung ist, die ethnische Einheiten allererst entstehen lässt, aber auch Raum für deren innere Komplexität und Heterogenität schafft. ${ }^{9}$ Und schließlich ließen die Forschungen zu Gender und Leiblichkeit deutlich werden, dass es weniger ein substanzieller Kern vermeintlich natürlicher Körperlichkeit, sondern die Logik kultureller Differenz ist, die das Geschlecht und den Körper formt. ${ }^{10}$ Die Fokussierung auf Verschiedenheit, Besonderheit und Abgrenzung führte also zu weitgehenden Erkenntnissen. Sie brachte jedoch auch eine starke, wenn nicht zu starke Akzentuierung von kultureller Differenz mit sich, womit Momente der Gleichheit oder Ähnlichkeit ausgeblendet wurden. Mit Blick auf negative Konsequenzen einer solchen Überbetonung gilt es, Differenz als relationale Differenz zu verstehen, als Verschiedenheit in Relation zu Gleichheit und Ähnlichkeit. ${ }^{11}$

Wenn nun aber (relationale) Differenz in all diesen ethnologischen Forschungsfeldern nicht als gegebenes Faktum anzusehen ist, sondern als formendes Prinzip soziokultureller Strukturierung, so muss die Frage nach der Macht gestellt werden. Begreifen wir Macht als das Vermögen, Objektivität zu setzen und durchzusetzen, und Differenz als formendes Prinzip, das Dinge voneinander unterscheidbar macht und dennoch in einer Beziehung zueinander hält, so kann diese Frage genauer gefasst werden: Die Form macht Differenz, aber jeder Form ist eine Logik der Differenzierung eigen, derer sich die Macht bedient, um die soziokulturelle Ordnung als Objektivität zu setzen. Und indem die Subjekte es sind, die in und durch ihre formende Praxis Objektivität differenzierend hervorbringen, werden diese selbst der relationalen Differenz unterworfen.

\section{Form, Macht und Differenz in den Forschungen von Brigitta Hauser-Schäublin}

Der analytische Blick auf Form, Macht und Differenz in ihren je spezifischen Relationen zieht sich durch die Forschungen von Brigitta Hauser-Schäublin. Er findet sich in 


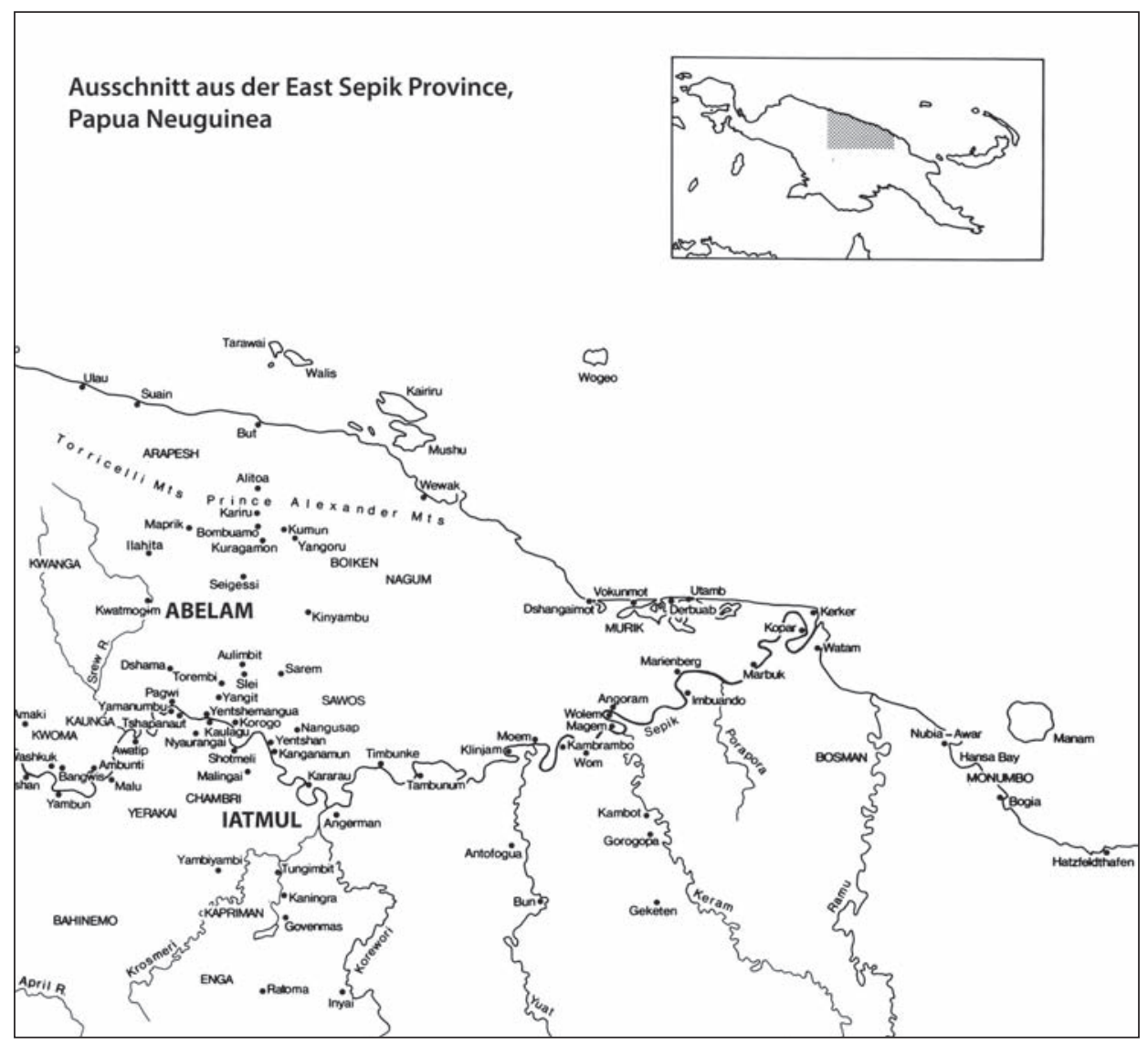

Forschungsgebiete von Brigitta Hauser-Schäublin in Papua-Neuguinea bei den Iatmul und Abelam.

ihren Ethnographien über die Iatmul und die Abelam in Papua-Neuguinea, in ihren Forschungen über Bali sowie in ihren Untersuchungen kultureller Diskurse und Praktiken in Mitteleuropa. So vielfältig die Regionen, so groß ist die Bandbreite ihrer thematischen Schwerpunkte, die von Museumsethnologie und materieller Kultur (z. B. 1988, 1989a, 2007c und im Druck) über Räumlichkeit (z. B. 1989b, 1992, 1997b, 2004, Dickhardt und Hauser-Schäublin 2003), Politik, Religion und Ritual (z. B. 1997b, 2003b, 2005a, 2005b, 2008, Hauser-Schäublin und Ardika 2008b), Gender und Körper (z. B. 1977, 1991, 2007a, 2007b, Hauser-Schäublin, Kalitzkus, Petersen und Schröder 2008, Hauser-Schäublin und Röttger-Rössler 1998) bis zu Cultural Heritage (z. B. HauserSchäublin und Krüger 2006) reichen und auch die allgemeinen Themen der Ethnologie wie Feldforschung (z. B. 2002, 2003a), Globalisierung (z. B. Hauser-Schäublin und Braukämper 2002) und Kulturkritik (z. B. 1997a) nie aussparen. Ein in seiner Breite und Tiefe so umfangreiches Werk kann an dieser Stelle nicht umfassend dargestellt 
werden. Hier sollen daher an drei Beispielen die Relationen von Form, Macht und Differenz in den Arbeiten von Brigitta Hauser-Schäublin umrissen werden.

Beginnen wir mit dem Verhältnis von Form und Differenz in dem, was die Arbeit Brigitta Hauser-Schäublins durchgängig in allen thematischen Feldern prägt: ihre konsequent durchgehaltene ethnologische Perspektive. Als eines der grundlegenden Momente einer solchen Perspektive wurde oben die Anerkennung der Differenz kultureller und gesellschaftlicher Formen als Ausgangspunkt des verstehenden Erklärens hervorgehoben. Nun zeigt Brigitta Hauser-Schäublins Analyse von Übergangsriten für Frauen bei den Abelam und den Iatmul im Sepik-Gebiet Neuguineas beispielhaft, wie wichtig eine Perspektive für das Verstehen kultureller Formen ist, die kulturelle Differenz nicht bloß vage postuliert, sondern in ihren konkreten kulturellen Ausformungen deutlich herauszuarbeiten vermag. In ihrem Artikel Puberty Rites, Women's naven, and Initiation (Hauser-Schäublin 1995) beschäftigt sie sich mit den unterschiedlichen Übergangsriten der Frauen in diesen beiden Gesellschaften, die zu den Sepik-Gesellschaften gezählt werden, eine Klassifizierung, die auf der Annahme kulturhistorisch begründeter Gemeinsamkeiten aufbaut. Wie aber sind dann die erheblichen Unterschiede in Inhalt, Funktion und Bedeutung zu erklären, die ein Vergleich der beiden Gesellschaften hinsichtlich der weiblichen Übergangsriten zutage bringt? Während bei den Abelamfrauen vor allem die Erstmenstruation rituell begleitet und als ein eigenständiger, von Frauen ausgeführter Übergangsritus ausgeformt wird, finden sich bei den Iatmulfrauen Übergangsriten anderer Art: Zum einen ist die Markierung kultureller Errungenschaften im Lebenslauf einer Frau im Kontext verwandtschaftlicher Beziehungen Anlass für ein naven genanntes Ritual, und zum anderen werden für einzelne Frauen Initiationen in bestimmte Positionen in den von Männern kontrollierten Klanstrukturen und den damit verbundenen Systemen esoterischen Wissens durchgeführt. Weder die Annahme eines gemeinsamen kulturellen Kontextes der ,Sepik-Gesellschaften', noch die in beiden Gesellschaften überaus große Wichtigkeit der Frauen für die Subsistenz, noch ein allgemeiner Begriff der Initiation können an und für sich diese Rituale und deren soziokulturelle Bedeutung in ihren Unterschiedlichkeiten verständlich machen. Erst ein differenzierender Blick auf die konkrete symbolische Ausformung der Rolle der Frauen in den Bereichen des Anbaus, der Fruchtbarkeit und Fortpflanzung sowie der Subsistenz ermöglicht ein Verstehen dieser signifikanten Unterschiede. Diese ergeben sich nämlich weder aus der homologen Ausformung eines gemeinsamen kulturellen Substrats, wie es der Begriff der ,Sepik-Gesellschaften' als gemeinsamer Bezugspunkt der Abelam und Iatmul nahelegt, noch aus der Ausformung einer allgemeinen rituellen Form der Initiation. Vielmehr müssen die konkreten Formen der weiblichen Übergangsriten und die darin zum Ausdruck kommenden unterschiedlichen Inhalte, Funktionen und Bedeutungen verstanden werden vor dem Hintergrund der konkreten Modi der Subsistenz, die sich bei den beiden Gesellschaften deutlich voneinander unterscheiden, und der symbolischen Ausformungen von Vorstellungen zur Fruchtbarkeit und zu Zeugungskräften im Zusammenhang mit diesen Modi der 


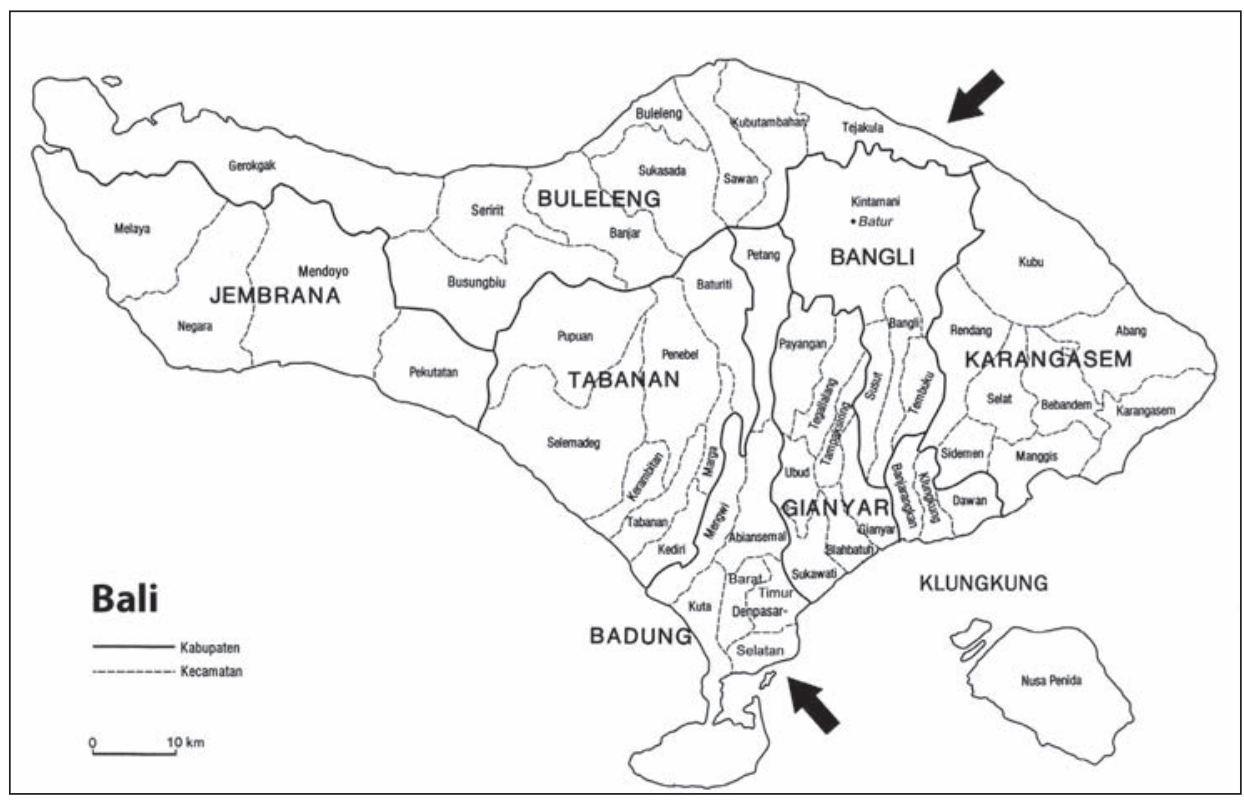

Forschungsgebiete von Brigitta Hauser-Schäublin in Bali.

Subsistenz (Hauser-Schäublin 1995:33-34 und 52). Kulturelle Differenz im Bemühen um ein Verstehen kultureller Formen ernst zu nehmen, heißt also, nicht an der Oberfläche der Formen zu verbleiben, sondern sie in ihren inneren Fügungen eingehend zu analysieren, heißt also, die oberflächliche Differenz nicht mit wohlfeilen Konstrukten wie ,Sepik-Gesellschaften' oder ,Initiation“ als eine oberflächliche wegzudefinieren, sondern der Differenz in ihrer tiefen Begründung in der konkreten Praxis Geltung zu verschaffen.

Eine spezifische Relation von Macht und Form thematisiert Brigitta Hauser-Schäublin in ihrem Aufsatz The Precolonial Balinese State Reconsidered (2003b), in dem sie Theorien der Staatenbildung mit einer eingehenden quellenkritischen Analyse unterschiedlicher Textgenres aus verschiedenen Jahrhunderten verbindet, um die konkrete Ausformung von vorkolonialer königlicher Macht auf Bali neu zu deuten. Ausgangspunkt ist die klassische Frage der Staatenbildung, ob Regionen, die sich durch Bewässerungssysteme auszeichnen, eine starke zentrale politische Macht benötigen. Marx und Wittfogel haben diese Frage bejaht - hier seien die Stichworte ,asiatische Produktionsweise“ respektive „orientalischer Despotismus“ genannt - und auf die Bedeutung des instrumentellen Aspekts von Macht zur Kontrolle der Ressource Wasser verwiesen. Für Bali haben Clifford Geertz mit der These des theater state und Stephen Lansing mit dem democratic irrigation model diese Frage jedoch aus unterschiedlichen Perspektiven verneint und mit dem empirisch unterfütterten Argument, dass die vorkolonialen balinesischen Könige keine Kontrolle über die Bewässerungssysteme gehabt hätten, gleich- 
sam die Absenz des instrumentellen Aspekts von Macht und damit einen schwachen Staat konstatiert. Brigitta Hauser-Schäublin setzt sich in ihrem Aufsatz kritisch mit Geertz und Lansing auseinander und fragt erneut, in welcher Form die balinesischen Könige nun Macht über die Bewässerungssysteme ausübten und wie die vorkolonialen balinesischen Königtümer vor diesem Hintergrund als politische Gebilde zu verstehen sind. Verzichteten sie mit der bloßen rituellen Inszenierung königlicher Macht in Form des theater state auf konkrete instrumentelle Macht in Form der bürokratisch organisierten Verfügungsgewalt über die Bewässerungssysteme - und damit über den ökonomisch existentiellen Bereich, den Nassreisanbau? Lassen sich die Bewässerungssysteme damit als eine nicht-hierarchisch organisierte Form der Ressourcenverteilung jenseits der Kontrolle durch den König, als democratic irrigation model verstehen? Mit anderen Worten: Existierten die balinesischen Königtümer gleichsam als Form ohne Macht bzw. fielen Form und Macht in eins?

Brigitta Hauser-Schäublins Interpretationen von vorkolonialen balinesischen Quellen sowie kolonialen Aufzeichnungen zeigen im Unterschied zu Geertz und Lansing, dass die Kontrolle der Bewässerungssysteme und die königliche Macht keinesfalls zwei nur lose miteinander verbundene Bereiche waren, in denen gar die bloße Inszenierung königlicher Macht eine konkrete ökonomische und politische Macht überflüssig machte. Ihre Studien ergaben, „,...] that temple and ritual networks creating a series of purposeful, fixed in time, cyclically repeating [...] migrations to different sites within a large area were a fundamental principle of organization of the precolonial, Balinese state and thus of royal supremacy" (2003b:156-157).

Diese These eröffnet gleich zwei neue Perspektiven auf die Relation von Macht und Form im balinesischen Kontext: Zum einen zeigt ihr sorgfältiges Studium der alten Quellen, dass die Macht balinesischer Könige durchaus instrumentelle Aspekte aufwies, direkte Verfügungsgewalt über die ökonomischen Ressourcen - konkreter über das Wasser und die Bewässerungssysteme - bedeutete und zudem eng mit den hierarchisch hoch stehenden Tempeln assoziiert war. Zum anderen wird deutlich, dass die spezifische Form des vorkolonialen Staates - und hier eben auch die Form im Sinne der inneren Fügung - untrennbar verbunden ist mit einer Macht, die er nicht nur instrumentell ausübt, sondern die ihn in und durch die Form von Tempelnetzwerken, zyklischen Ritualen, Pilgerfahrten und ökonomischen Verpflichtungen prozesshaft in seiner besonderen Form hervorbringt (Hauser-Schäublin 2003b:157). Die Macht des Königs definierte sich dabei primär über die Fähigkeit zur Mobilisierung großer Menschenmassen. ${ }^{12}$ Macht wird auf kulturell höchst spezifische Art und Weise immer wieder gemacht und erinnert in dieser fluiden Form nur sehr peripher an den territorial definierten Staat europäischer Provenienz.

Aktuell beschäftigt sich Brigitta Hauser-Schäublin mit dem Thema cultural property aus interdisziplinärer Perspektive. ${ }^{13}$ Auch hier lassen sich spezifische Konfigurationen von Form, Macht und Differenz finden. Differenz ist dabei ein konstitutives Moment für cultural property, das sich als kulturell ganz spezifisch Eigenes, Typisches, 
Besonderes von allem anderen abgrenzt. Differenz ist somit weniger eine Eigenschaft als ein relationales Verhältnis. Dieses Eigene, Typische, Besondere eines cultural property existiert mit einer spezifischen äußeren Form und - als Ausdruck des kulturell Eigenen - mit einer spezifischen inneren Fügung. Cultural property lässt sich jedoch nicht auf eine unschuldige Authentizität reduzieren, sondern nur im Kontext konkreter Machtbeziehungen verstehen, ohne darin aufzugehen. Wessen kulturell Eigenes wird - sowohl in Konkurrenz von Gesellschaften untereinander als auch innerhalb einer ja immer heterogenen Gesellschaft - zum cultural property? Welche Formen von cultural property bringt der globale Diskurs über den Schutz materieller wie immaterieller kultureller Phänomene hervor, welche der nationale Wunsch nach der Präsentation kulturellen Reichtums? Wie verändert sich in diesem Kontext das Verständnis für und von Kultur insgesamt, wie die soziale Praxis? Wie verschiebt sich Macht im Sinne der Verfügungsgewalt im Prozess der Zertifizierung von kulturellen Phänomenen etwa zu einem Weltkulturerbe? Die praktische Wirkmächtigkeit von Differenz, so lässt sich konstatieren, wird im Kontext von cultural heritage durch den Einsatz spezifischer kultureller Formen von verschiedenen Akteuren in machtstrukturierten Kontexten formuliert und beansprucht.

\section{Zu den Beiträgen in diesem Band}

Bei der Zusammenstellung dieses Buches haben wir versucht, die vielfältigen Motive, die uns in Arbeit und Werk Brigitta Hauser-Schäublins und in den Beiträgen entgegentraten, mit den soeben umrissenen Aspekten der Relation von Form, Macht und Differenz in Verbindung zu bringen. Der Begriff des Motivs erwies sich dabei für uns in seiner schillernden Mehrdeutigkeit als besonders inspirierend: Das Motiv als Beweggrund ethnologischen Forschens zwischen engagierter Kulturkritik und ethnographischer Faszination, als Gegenstand der Darstellung und Analyse sowie als Thema und Leitmotiv ethnologischen Forschens. Und so symbolisiert das Motiv unseres Titelbildes - der Blick durch das Tor eines balinesischen Tempels mit einem Hoheitsschirm und der roten Spitze einer Standarte als Hinweis auf eine dort anwesende Gottheit ${ }^{14}$ für uns in seiner ethnographischen Faszination einen Beweggrund ethnologischen Forschens und verweist zugleich in der Art, wie in ihm die Form die Macht zu verdecken und doch zugänglich zu machen scheint, auf ein Leitmotiv dieses Forschens. Auch in den Beiträgen entdeckten wir sehr unterschiedliche Motive, die sich aber unter verschiedenen Aspekten der Relation von Form, Macht und Differenz zusammenfügen ließen. Dabei wird, den oben skizzierten Relationen folgend, in den Kapiteln dieses Buches je ein Begriff in seinem Verhältnis zu den beiden anderen in den Mittelpunkt gestellt. Entsprechend nähern sich die Beiträge in diesem Buch der Komplexität dieser Relationen aus verschiedenen regionalen und thematischen Perspektiven. Je nach thematischem Fokus steht Form in Relation zu Macht und Differenz oder Macht in 
Relation zu Form und Differenz oder Differenz in Relation zu Macht und Form im Mittelpunkt der Betrachtung. Die Beiträge des letzten Kapitels reflektieren schließlich das ethnologische Forschen als Praxis von Form, Macht und Differenz. Da sich die Bedeutung der Beiträge der Kapitel zwei bis fünf für das Thema des Buches selbst erschließt, soll hier nur näher auf das erste Kapitel eingegangen werden.

Feldforschung als Praxis von Form, Macht und Differenz ist seit über 35 Jahren die große Leidenschaft von Brigitta Hauser-Schäublin, die sie auch theoretisch reflektiert hat (2002 u. 2003a). Dieser Leidenschaft in ihrer konkreten Form, der komplexen Erfahrung, „[...] was das Kulturwesen Mensch, der/die Andere ,ist ${ }^{\star}$ und was ich selber bin" (Hauser-Schäublin 2002:73), ist das erste Kapitel gewidmet. Die Fotos von Jörg Hauser zeigen diese Begegnung zwischen dem eigenen und dem relational fremden Sein als Kulturwesen Mensch. Feldforschung als eminent sinnliche Praxis und Erfahrung findet sich in der bloßen textuellen Repräsentation nur unzureichend wieder. In den Fotografien, die Brigitta Hauser-Schäublin an den Orten ihrer Forschungen im Gespräch oder in Aktion mit anderen zeigen, wird jedoch etwas von dem lebendig, was ein Text nur schwer wiedergeben kann: die konkrete, alltägliche Praxis des Forschens, die Momente von Nähe und Verstehen, von Fremdheit, von Mühsal, von Ruhe und Entspannung und immer wieder Momente des intensiven Zuhörens und des Dialogs. Bei genauerer Betrachtung der Bilder zeigt sich, dass Form, Macht und Differenz auch visuell vermittelt werden: durch Körperhaltungen und Gesten, durch die räumliche Struktur, durch Blicke und Positionierungen, durch Sitzordnungen und Kleidung. Gleichzeitig legen sie Zeugnis ab von einer jahrzehntelangen vertrauten und selbstverständlichen Zusammenarbeit zwischen Brigitta Hauser-Schäublin und Jörg Hauser sowie von seiner Meisterschaft als Fotograf.

Die Fotos werden ergänzt durch Interviews mit I Wayan Terang aus Julah, Jero Guru Nengah Teket aus Batur und Jero Mangku Dalem I Nyoman Sutarmi aus Sembiran, drei Forschungspartnern von Brigitta Hauser-Schäublin, die im Kontext ihrer Forschungen auf Bali von zentraler Bedeutung sind. Mit den Interviews sollen ihre balinesischen Wegbegleiter einen angemessenen Platz in dieser Festschrift finden und Brigitta Hauser-Schäublins Leidenschaft für Feldforschung, die sich in ganz existentieller Hinsicht aus ihrer Neugier auf Menschen und ihrer Faszination durch Begegnungen und Beziehungen speist, gewürdigt werden:

Bei jedem Forschungsaufenthalt bin ich wieder verzaubert von den Geweben der Beziehungen der Menschen dort, aber auch denjenigen zwischen mir und ihnen, von der Begegnung mit Menschen, mit denen mich z. T. seit langem vieles verbindet, aber auch vieles trennt. (Hauser-Schäublin 2002:73)

Die drei Interviews bieten nicht nur einen sehr lebendigen und humorvollen Einblick in die Praxis der Feldforschung, die Bedeutung jahrelanger Beziehungen und die Persönlichkeit der drei Interviewten, sondern zeigen gerade in ihrer Kombination auch sehr klar, wie sehr die eigene Perspektivität - wie eingangs erläutert - durch Prozesse 
Michael Dickhardt, Karin Klenke und Elfriede Hermann

der Differenzierung auf der Basis von Geschlecht, Alter und Macht, aber auch auf der Basis der kulturellen Herkunft in der Feldforschung bestimmt wird und zu einer konkreten relationalen Positionierung im Feld führt, wie Brigitta Hauser-Schäublin es sehr differenziert am Beispiel ihrer eigenen Feldforschungserfahrungen darstellt (2002). Alter und Status der eigenen Person strukturieren, so zeigen die Interviews, das jeweilige Verhältnis zu Brigitta Hauser-Schäublin in einer in Bezug auf Form, Macht und Differenz je spezifischen Weise: Für I Wayan Terang, dem jüngsten der drei, sind sie und ihr Mann „wie Eltern“, denen er dankbar für ihre Tipps zu seiner Amtsführung als Bürgermeister und für ihre materielle Unterstützung ist. Jero Mangku Dalem I Nyoman Sutarmi, ein Tempelpriester, der etwa im gleichen Alter wie Brigitta Hauser-Schäublin ist, beschreibt ihr Verhältnis dagegen als ebenbürtig - so ebenbürtig, dass selbst die Dozenten der Universität Udayana erstaunt gewesen seien. Jero Guru Nengah Teket schließlich erläutert mit der ganzen Würde nicht nur seines Alters, sondern auch seines Amtes als Priester am Batur-Tempel, einem Tempel mit zentraler Bedeutung für ganz Bali, dass er sie als sein Kind ansehe und daher eine große - und schwere? - Verantwortung trage, da er dadurch verpflichtet sei, sie zu versorgen und ihr weiterzuhelfen. Gleichzeitig wird darin deutlich, dass sowohl die jeweiligen Verhältnisse selbst als auch ihre sprachlichen Explikationen bestimmten kulturellen Formen folgen, in denen Respekt für Ältere und sozial höher Stehende unter anderem durch (rhetorische) Bescheidenheit zum Ausdruck gebracht wird. So erscheint in Wayang Terangs Erzählungen sein Verhältnis zu Brigitta Hauser-Schäublin als ein einseitiges, in dem ausschließlich er der Empfangende ist und seine eigene Arbeit, die er für sie leistet, völlig in den Hintergrund tritt. Ihr verdankt er, so fasst er ihre gemeinsame Arbeit zusammen, letztlich sein Amt als Bürgermeister und sogar einen Besuch beim Präsidenten, sein gesamtes Dorf verdankt ihr die positive Entwicklung. Jero Mangku Dalem dagegen erläutert, dass ihr Verhältnis auf Gegenseitigkeit beruhe: „Unser Leben besteht doch daraus, dass wir uns gegenseitig helfen!"Jero Guru Nengah Teket wiederum, als Priester des Batur-Tempels der Ranghöchste der drei, stellt klar, dass ihre gemeinsame Arbeit nicht auf Gegenseitigkeit beruht, sondern er in diesem Verhältnis der ausschließlich Gebende ist:

Was auch immer sie gefragt hat, konnte ich beantworten. Alles, was ich schon wusste, musste sie dann nicht mehr aus irgendwelchen Texten lernen. Von ihr konnte ich nichts Neues mehr lernen. Sie hat ihre Ergebnisse nur immer mit mir abgeglichen.

Alle drei thematisieren sehr klar diese relationalen Positionierungen und deren Implikationen. Form, Macht und Differenz erfahren hier kulturell spezifische und pragmatische Artikulierungen. Nicht zuletzt zeigen diese Interviews jedoch auch jenseits aller Überlegungen zu Form, Macht und Differenz die große Wertschätzung, die Brigitta Hauser-Schäublin von ihren balinesischen Informanten erfährt. Das letzte Wort soll hier I Wayan Terang erhalten: 
Ich denke, der spezielle Erfolg von Ibu Brigittas Forschung lässt sich auf jeden Fall darauf zurückführen, dass sie immer direkt auf die Menschen zugegangen ist und ihr das persönliche Kennenlernen wichtig war, so dass die Bevölkerung sie sehr geschätzt hat.

\section{Anmerkungen}

1 Die folgenden Überlegungen verstehen sich hinsichtlich des Formbegriffs vor allem vor dem Hintergrund des Ansatzes Ernst Cassirers, dessen Formbegriff auf der Grundlage der strukturierungstheoretischen Entwürfe Bourdieus, der von strukturierenden Strukturen spricht (1987:98), und Giddens, der auf den dualen Charakter der Struktur verweist (1989:XXXI,242). Zusammenfassendes findet sich bei Bourdieu (1987:97-121, 1983 und 1998) sowie bei Giddens (1989:1-40). Zum Formbegriff Cassirers siehe Cassirer (1956, 1992 und 1994, vor allem Band 1:3-52). Siehe dazu auch Dickhardt (2001:27-58). Die Überlegungen zum Machtbegriff nehmen ihren Ausgang bei Michel Foucault und dessen Denken zum Begriff der Macht (1976, 1991 und 1995) und Pierre Bourdieu (vor allem 1987:97-179). Zusammenfassendes zu Foucault findet sich bei Lorey (1999) und Ricken (2004). Für die Überlegungen zum Begriff der Differenz in seinen epistemologischen und methodologischen Dimensionen sei auf die konzise Zusammenfassung von Moore und Sanders (2006:1-3) sowie auf Rottenburg (2006) verwiesen. Grundlegend sind freilich Moore (1994) und Wagner (1981). Für differenzierte und kritische Positionen sei verwiesen auf Abu-Lughod, die vor einer ,anthropology's complicity in the reification of cultural difference" (Abu-Lughod 2002:783) warnt, und auf Hermann (2006).

2 Um der Lesbarkeit willen wird auf die jeweilige Nennung der weiblichen und männlichen Form verzichtet, ohne das nicht genannte Geschlecht dabei ausklammern zu wollen.

3 Siehe dazu Anmerkung 1.

4 Siehe dazu Anmerkung 1.

5 Siehe dazu Anmerkung 1.

6 Diese Hinwendung zur kulturellen Differenz kann als Geburtsstunde der heutigen Ethnologie in Abwendung vom klassischen Evolutionismus verstanden werden, wie sie Franz Boas und seine Schüler und Schülerinnen vollzogen haben; dazu siehe Hatch (1983, vor allem die Seiten 24-26 und 52-59).

7 Der Weg zu dieser Erkenntnis war ein langer und gewundener, maßgeblich bestimmt von der Writing Culture-Debatte, der postmodernistischen Kritik und der Kritik an den Konsequenzen einer homogenisierenden sowie einseitig kulturalistischen und relativistischen Kulturanthropologie. Dazu siehe z. B. Abu-Lughod (1991, 1993 und 2002), Bloch (2005) sowie Clifford und Marcus (1986).

8 Dazu zusammenfassend Fuchs und Berg (1999) und Hauser-Schäublin (2002).

9 Dazu siehe Barth (1969), Fenton (2003), Jenkins (1997) und Linnekin und Poyer (1990).

10 Dazu siehe z. B. Hauser-Schäublin und Röttger-Rössler (1998) und Moore (1994).

11 Dazu siehe Hermann (2006).

12 Wurden während des Suharto-Regimes Religion und Politik strikt getrennt, knüpfen heute in der Zeit der politischen Dezentralisierung Indonesiens balinesische Politiker an diese Traditionen an - ein Thema, das Brigitta Hauser-Schäublin aktuell untersucht.

13 Seit 2008 ist sie Mitglied der DFG-geförderten Forschergruppe „Die Konstituierung von Cultural Property. Akteure, Diskurse, Kontexte, Regeln“ und arbeitet mit VölkerrechtlerIn- 
nen zu den Prozessen, die dazu führten, dass die an der thailändisch-kambodschanischen Grenze gelegene Tempelanlage Preah Vihear zum UNESCO-Weltkulturerbe ernannt wurde, sowie zu den Grenzsstreitigkeiten, die der Ernennung folgten. Damit bleibt sie ihrer Leidenschaft für interdisziplinäres Arbeiten sowohl in der Forschung als auch in der Lehre treu. Neben der fruchtbaren Zusammenarbeit mit der Archäologie und der Geographie im Kontext ihrer Arbeiten zu Bali hat sie in der Lehre vielfach interdisziplinäre Lehrveranstaltungen mit VertreterInnen anderer Fächer der Universität Göttingen angeboten.

14 Für das Titelbild danken wir Jörg Hauser herzlichst!

\section{Literatur}

Abu-Lughod, Lila (1991) Writing Against Culture. In: Richard G. Fox (Hg.): Recapturing Anthroplogy. Working in the Present, S. 137-162. Santa Fe: School of American Research Press.

Abu-Lughod, Lila (1993) Writing Women's Worlds. Bedouin Stories. Berkeley: University of California Press.

Abu-Lughod, Lila (2002) Do Muslim Women Really Need Saving? Anthropological Reflections on Cultural Relativism and Its Others. In: American Anthropologist 104 (3), S. 783-790.

Barth, Fredrik (1969) Introduction. In: Fredrik Barth (Hg.): Ethnic Groups and Boundaries. The Social Organization of Culture Difference, S.9-38. Oslo: Univeritets Forlaget.

Bloch, Maurice (2005) Where Did Anthropology Go? Or the Need for ,Human Nature'. In: Maurice Bloch: Essays on Cultural Transmission, S. 1-19. Oxford: Berg (London School of Economics Monographs on Social Anthropology 75).

Bourdieu, Pierre (1983) Zur Soziologie der symbolischen Formen. Frankfurt a.M.: Suhrkamp (stw 107).

Bourdieu, Pierre (1987) Sozialer Sinn. Kritik der theoretischen Vernunft. Frankfurt a.M.: Suhrkamp.

Bourdieu, Pierre (1998) Praktische Vernunft. Zur Theorie des Handelns. Frankfurt a.M.: Suhrkamp (Edition Suhrkamp NF 985).

Cassirer, Ernst (1956) Der Begriff der symbolischen Form im Aufbau der Geisteswissenschaften. In: Ernst Cassirer: Wesen und Wirkung des Symbolbegriffs, S. 169-200. Darmstadt: Wissenschaftliche Buchgesellschaft.

Cassirer, Ernst (1992) An Essay on Man. New Haven: Yale University Press.

Cassirer, Ernst (1994) Philosophie der symbolischen Formen, 3 Bände. Darmstadt: Wissenschaftliche Buchgesellschaft. 
Clifford, James und George E. Marcus (Hg.) (1986) Writing Culture. The Poetics and Politics of Ethnography. Berkeley: University of California Press.

Dickhardt, Michael (2001) Das Räumliche des Kulturellen. Entwurf zu einer kulturanthropologischen Raumtheorie am Beispiel Fiji. Münster: Lit (Göttinger Studien zur Ethnologie 7).

Dickhardt, Michael und Brigitta Hauser-Schäublin (2003) Eine Theorie kultureller Räumlichkeit als Deutungsrahmen. In: Brigitta Hauser-Schäublin und Michael Dickhardt (Hg.): Kulturelle Räume - räumliche Kultur. Zur Neubestimmung des Verbältnisses zweier fundamentaler Kategorien menschlicher Praxis, S. 13-42. Münster: Lit (Göttinger Studien zur Ethnologie 10).

Fenton, Steve (2003) Ethnicity. Cambridge: Polity.

Foucault, Michel (1976) Die Macht und die Norm. In: Michel Foucault (Hg.): Mikrophysik der Macht. Michel Foucault über Strafjustiz, Psychiatrie und Medizin, S. 114123. Berlin: Merve Verlag.

Foucault, Michel (1991) Die Ordnung des Diskurses. Frankfurt a.M.: Fischer.

Foucault, Michel (1995) Überwachen und Strafen. Die Geburt des Gefängnisses. Frankfurt a.M.: Suhrkamp (stw 184).

Fuchs, Martin und Eberhard Berg (1999) Phänomenologie der Differenz. Reflexionsstufen ethnographischer Repräsentation. In: Eberhard Berg und Martin Fuchs (Hg.): Kultur, soziale Praxis, Text. Die Krise der ethnographischen Repräsentation, S. 11-108. Frankfurt a.M.: Suhrkamp (stw 1051).

Giddens, Anthony (1989) The Constitution of Society. Outline of the Theory of Structuration. Cambridge: Polity Press.

Hatch, Elvin (1983) Culture and Morality. The Relativity of Values in Anthropology. New York: Columbia University Press.

Hauser-Schäublin, Brigitta (1977) Frauen in Kararau. Zur Rolle der Frau bei den Iatmul am Mittelsepik, Papua New Guinea. Basel: Ethnologisches Seminar und Museum für Völkerkunde (Basler Beiträge zur Ethnologie 18).

Hauser-Schäublin, Brigitta (1988) Die Rolle der Völkerkundemuseen bei der Vermittlung von Fremdbildern. In: Ina-Maria Greverus, Konrad Köstlin und Heinz Schilling (Hg.): Kulturkontakt, Kulturkonflikt: zur Erfahrung des Fremden, Teil 1. (26. Deutscher Volkskundekongress in Frankfurt vom 28. September bis 2. Oktober 1987), S. 555-561. Frankfurt a.M.: Institut für Kulturanthropologie und Europäische Ethnologie (Notizen, Institut für Kulturanthropologie und Europäische Ethnologie, Universität Frankfurt a.M. 28). 
Michael Dickhardt, Karin Klenke und Elfriede Hermann

Hauser-Schäublin, Brigitta (1989a) Leben in Linie, Muster und Farbe. Einführung in die Betrachtung aussereuropäischer Kunst am Beispiel der Abelam, Papua-Neuginea. Basel: Birkhäuser.

Hauser-Schäublin, Brigitta (1989b) Kulthäuser in Nordnenguinea. Teil l: Architektur, Funktion und Symbolik des Kulthauses bei den Abelam. Teil 2: Vergleichende Studien zu Kulthäusern im Sepik-Gebiet und an der Nordküste. Berlin: Akademie-Verlag (Abhandlungen und Berichte des Staatlichen Museums für Völkerkunde Dresden 43; Monographien 7).

Hauser-Schäublin, Brigitta (Hg.) (1991) Ethnologische Frauenforschung. Ansätze, Methoden, Resultate. Berlin: Reimer.

Hauser-Schäublin, Brigitta (1992) Der verhüllte Schrein. Sakralarchitektur und ihre Umhüllungen in Bali. In: Wolfgang Marschall et al. (Hg.): Die fremde Form, S. 171-200. Bern: Société Suisse d'Ethnologie (Ethnologica Helvetica 16).

Hauser-Schäublin, Brigitta (1995) Puberty Rites, Women's Naven, and Initiation: Women's Rituals of Transition in Abelam and Iatmul Culture. In: Nancy Lutkehaus und Paul Roscoe (Hg.): Gender Rituals: Female Initiation in Melanesia, S.33-53. New York und London: Routledge.

Hauser-Schäublin, Brigitta (1997a) Blick zurück im Zorn. Ethnologie als Kulturkritik. Zeitschrift für Ethnologie 122:3-17.

Hauser-Schäublin, Brigitta (1997b) Traces of Gods and Men. Temples and Rituals as Landmarks of Social Events and Processes in a South Bali Village. Berlin: Reimer.

Hauser-Schäublin, Brigitta (2002) Gender: Verkörperte Feldforschung. In: Hans Fischer (Hg.): Feldforschungen: Erfahrungsberichte zur Einführung (2. Auflage, Neufassung), S.73-100. Berlin: Reimer.

Hauser-Schäublin, Brigitta (2003a) Teilnehmende Beobachtung. In: Bettina Beer (Hg.): Methoden und Techniken der Feldforschung, S.33-54. Berlin: Reimer.

Hauser-Schäublin, Brigitta (2003b) The Pre-colonial Balinese State Reconsidered: A Critical Evaluation of Theory Construction on the Relationship Between Irrigation, the State, and Ritual. Current Anthropology 44(2):153-182.

Hauser-Schäublin, Brigitta (2004) The Politics of Sacred Space: Using Conceptual Models of Space for Socio-political Transformations in Bali. Bijdragen tot de Taal-, Land-en Volkenkunde 160(2/3):283-314.

Hauser-Schäublin, Brigitta (2005a) On Irrigation and the Balinese State. Reply. Current Anthropology 46(2):306-308. 
Hauser-Schäublin, Brigitta (2005b) Temple and King: Resource Management, Rituals and Redistribution in Early Bali. Journal of the Royal Anthropological Institute 11(4):747-771.

Hauser-Schäublin, Brigitta (2007a) „Was die Europäer uns gebracht haben, ist der Körper“. Von der Undenkbarkeit des Körpers als Objekt. In: Jochen Taupitz (Hg.): Kommerzialisierung des menschlichen Körpers, S. 21-36. Berlin: Springer.

Hauser-Schäublin, Brigitta (2007b) Blutsverwandtschaft. In: Christina von Braun und Christoph Wulf (Hg.): Mythen des Blutes. Publikation der gleichnamigen Tagung vom 1.-3. Dezember 2005 in Berlin, S. 171-183. Frankfurt a.M.: Campus Verlag.

Hauser-Schäublin, Brigitta (2007c) Weiss in einem Meer von Rot. Die bemalte Giebelwand des Abelam-Kulthauses. In: Anna Schmid und Alexander Brust (Hg.): Rot. Wenn Farbe zur Täterin wird (Buch zur Ausstellung im Museum der Kulturen, Basel), S. 190-194. Basel: Christoph Merian Verlag.

Hauser-Schäublin, Brigitta (2008) Ritual, Pilgrimage and the Reconfiguration of the State. Sacred Journeys in the Political Landscape of Bali (Indonesia). In: Jörg Gengnagel, Monika Horstmann und Gerald Schwedler (Hg.): Prozessionen, Wallfahrten, Aufmärsche. Bewegung zwischen Religion und Politik in Europa und Asien seit dem Mittelalter, S.288-311. Köln: Böhlau.

Hauser-Schäublin, Brigitta (Im Druck) Changing Contexts, Shifting Meanings: The Cook/Forster Collection, for Example. In: Elfriede Hermann (Hg.): Changing Contexts - Shifting Meanings: Transformations of Cultural Traditions in Oceania. Honolulu: The University of Hawai'i Press.

Hauser-Schäublin, Brigitta und I Wayan Ardika (Hg.) (2008) Burials, Texts and Rituals. Ethnoarchaeological Investigations in North Bali, Indonesia. Göttingen: Universitätsverlag Göttingen (Göttinger Beiträge zur Ethnologie 1).

Hauser-Schäublin, Brigitta und Ulrich Braukämper (2002) Einleitung: zu einer Ethnologie der weltweiten Verflechtungen. In: Brigitta Hauser-Schäublin und Ulrich Braukämper (Hg.): Ethnologie der Globalisierung. Perspektiven kultureller Verflechtungen, S.9-14. Berlin: Reimer.

Hauser-Schäublin, Brigitta, Vera Kalitzkus, Imme Petersen und Iris Schöder (2008) Der geteilte Leib: die kulturelle Dimension von Organtransplantation und Reproduktionsmedizin in Deutschland. Überarbeitete Version (2005) und Ergänzung (2008) des gleichnamigen Buches von 2001. Verfügbar unter <http://webdoc.sub.gwdg. $\mathrm{de} / \mathrm{pub} / \mathrm{mon} / 2008 /$ hauser-schaeublin.pdf $>$.

Hauser-Schäublin, Brigitta und Gundolf Krüger (2006) Pacific Cultural Heritage The Göttingen Cook/Forster Collection. In: Therese Weber und Jeanie Watson 
(Hg.): Cook's Pacific Encounters: The Cook-Forster-Collection of the Georg-August University of Göttingen, S. 15-28. Canberra: National Museum of Australia Press.

Hauser-Schäublin, Brigitta und Birgitt Röttger-Rössler (1998) Differenz und Geschlecht - Eine Einleitung. In: Brigitta Hauser-Schäublin und Birgitt RöttgerRössler (Hg.): Differenz und Geschlecht. Neue Ansätze in der ethnologischen Forschung, S.7-22. Berlin: Reimer.

Hermann, Elfriede (2006) Korrelationen von Verschiedensein und Gleichsein als Ko-Differenz: Selbst und Ethnie bei den Banabans in Fiji (Universität Göttingen: unveröffentlichte Habilitationsschrift).

Jenkins, Richard (Hg.) (1997) Rethinking Ethnicity. Arguments and Explorations. London: Sage.

Linnekin, Jocelyn und Lin Poyer (Hg.) (1990) Cultural Identity and Ethnicity in the Pacific. Honolulu: University of Hawaii i Press.

Lorey, Isabell (1999) Macht und Diskurs bei Foucault. In: Hannelore Bublitz (Hg.): Das Wuchern der Diskurse. Frankfurt a.M.: Campus.

Moore, Henrietta (1994) A Passion for Difference. Essays in Anthropology and Gender. Camebridge: Polity.

Moore, Henrietta und Todd Sanders (2006) Anthropology and Epistemology. In: Henrietta Moore und Todd Sanders (Hg.): Anthropology in Theory. Issues in Epistemology, S. 1-21. Malden: Blackwell.

Ricken, Norbert (2004) Die Macht der Macht - Rückfragen an Michel Foucault. In: Norbert Ricken und Markus Rieger-Ladich (Hg.): Michel Foucault: Pädagogische Lektüren, S. 119-143. Wiesbaden: VS Verlag.

Rottenburg, Richard (2006) Untrivializing Difference. A Personal Introduction. In: Richard Rottenburg, Burkhard Schnepel und Shingo Shimada (Hg.): The Making and Unmaking of Differences. Anthropological, Sociological and Philosophical Perspectives), S.7-12. Bielefeld: transcript (Culture and Social Practice).

Wagner, Roy (1981) The Invention of Culture. Chicago: University of Chicago Press. 